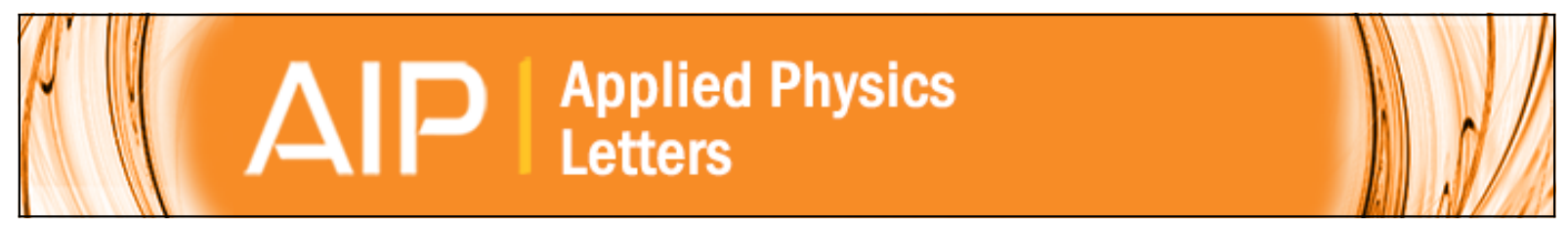

\title{
Adsorption-induced conversion of the carbon nanotube field effect transistor from ambipolar to unipolar behavior
}

Donghun Kang, Noejung Park, Jaewoong Hyun, Eunju Bae, Juhye Ko, Jujin Kim, and Wanjun Park

Citation: Applied Physics Letters 86, 093105 (2005); doi: 10.1063/1.1869548

View online: http://dx.doi.org/10.1063/1.1869548

View Table of Contents: http://scitation.aip.org/content/aip/journal/apl/86/9?ver=pdfcov

Published by the AIP Publishing

\section{Articles you may be interested in}

Hysteretic transfer characteristics of double-walled and single-walled carbon nanotube field-effect transistors Appl. Phys. Lett. 91, 143118 (2007); 10.1063/1.2789789

Room-temperature single charge sensitivity in carbon nanotube field-effect transistors Appl. Phys. Lett. 89, 243502 (2006); 10.1063/1.2399942

Ab initio study of the effect of water adsorption on the carbon nanotube field-effect transistor Appl. Phys. Lett. 89, 243110 (2006); 10.1063/1.2397543

Prospect of cobalt-mix-tetraethoxysilane method on localized lateral growth of carbon nanotubes for both $p$ - and $\mathrm{n}$-type field effect transistors

J. Vac. Sci. Technol. B 24, 2282 (2006); 10.1116/1.2345207

Self-assembled carbon-nanotube-based field-effect transistors

Appl. Phys. Lett. 85, 5025 (2004); 10.1063/1.1823017

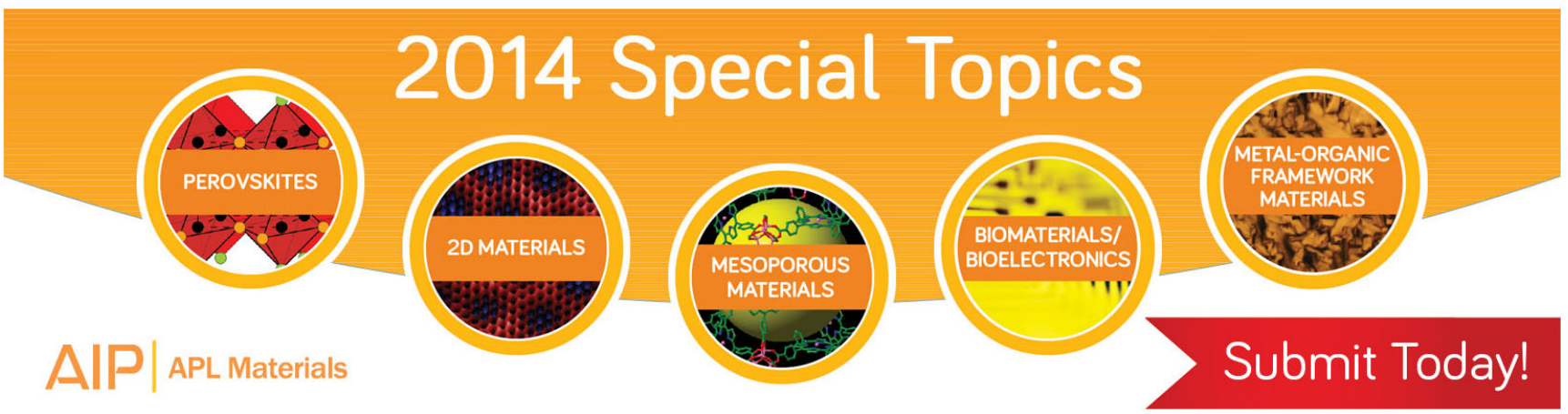




\title{
Adsorption-induced conversion of the carbon nanotube field effect transistor from ambipolar to unipolar behavior
}

\author{
Donghun Kang, Noejung Park, Jaewoong Hyun, Eunju Bae, Juhye Ko, Jujin Kim, ${ }^{\text {a) }}$ and \\ Wanjun Park ${ }^{\text {b) }}$ \\ Materials and Devices Lab., CSE Lab., Samsung Advanced Institute of Technology, Mt.14-1, Neongseo-Ri, \\ Giheung-Eup, Yongin City, Gyeonggi-Do 449-712, Korea
}

(Received 26 July 2004; accepted 18 January 2005; published online 23 February 2005)

\begin{abstract}
We investigate ambipolar to unipolar transition by the effect of ambient air on the carbon nanotube field-effect transistor. A unipolar transport property of the double-walled nanotube field-effect transistor and its conversion from ambipolar behavior are observed. We suggest that adsorptions of oxygen molecules, whose lowest-unoccupied-molecular-orbital state is around the midgap of the carbon nanotube, could suppress the electron channel formation and, consequently, result in the unipolar transport behavior. (C) 2005 American Institute of Physics. [DOI: 10.1063/1.1869548]
\end{abstract}

Since the discovery of carbon nanotubes (CNTs), ${ }^{1}$ their various aspects have been explored in different fields of science and technology. In particular, the unique onedimensional geometry and the excellent transport property have made CNTs the most promising building blocks for the next-generation electronic devices. ${ }^{2,3}$ The most important application in electronics is the CNT field effect transistor (FET). ${ }^{4}$ However ambipolar behavior in CNT-FET (Ref. 5) limits in the development of nanotube-based integrated circuit application by unexpected leakage current before the turn-on bias.

In this work, we show a unipolar transport property of the double-walled nanotube (DWNT) FET and its conversion from ambipolar behavior. We choose DWNT-FET to maximize ambipolar behavior ${ }^{6}$ since it is mainly ascribed to the smaller band gap arising from the large diameter of outer shell. Purified DWNTs were suspended in a solvent and spin coated on the $\mathrm{SiO}_{2}$ grown on heavily doped $p$-type $\mathrm{Si}$ substrate. Scanning probe microscopy locates individually separated nanotubes on the substrate. After generating patterns for metal contact by electron-beam lithography, metal electrodes with $100 \mathrm{~nm}$ Pd were defined by the lift-off process. Typical system resistances without gate bias were in the range of several hundreds of $\mathrm{k} \Omega$ to $\mathrm{M} \Omega$. Electrical measurement was carried out in a probe station chamber equipped with a pumping unit, which can pump down the chamber to $10^{-6}$ Torr.

In Fig. 1, we present the current-voltage $\left(I_{\mathrm{ds}}-V_{g}\right)$ curves of the DWNT-FET measured with various source and drain voltages $\left(V_{\mathrm{ds}}\right)$ under the influence of ambient air. A typical $p$-channel behavior is observed in our DWNT-FET. It could be described as a device operating in $p$-channel depletion mode, whose turn-off voltages are about $V_{g}=4 \mathrm{~V}$. With careful examination, it is observed that there are small bumps in the $I_{\mathrm{ds}}-V_{g}$ curves at about $3 \mathrm{~V}$ above the turn-off voltage. In the latter part, we discuss that the observed small bumps and traces of current in the positive side of gate voltage could indicate the suppression of electron channel formation.

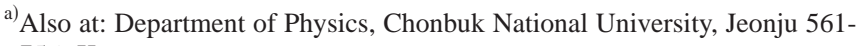
756, Korea.

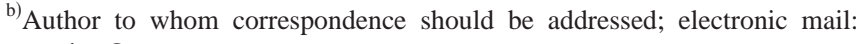
wanjun@ @amsung.com
}

We now turn to investigate the transfer characteristics of CNT-FET in different vacuum conditions. In Fig. 2, we plot the $I_{\mathrm{ds}}-V_{g}$ curves of DWNT-FET at several intermediate pumping stages from ambient air to vacuum of 3 $\times 10^{-6}$ Torr. The $n$-channel starts to emerge and the FET gradually shows an ambipolar behavior as it passes through the several pumping stages. After $72 \mathrm{~h}$ of pumping, we have a complete conversion of transport property of DWNT-FET from unipolar to ambipolar. In fact, the overall conversion process can be reversible and can reproduce the patterns that are shown in Fig. 2.

Heinze et $\mathrm{al}^{7}$ discussed that the work function variation in the electrode leads to an asymmetric change in the $I_{\mathrm{ds}}-V_{g}$ curve, inducing less current for one polarity of gate bias and more current for the opposite polarity under the influence of ambient air. Such a change has largely attributed to the oxygen effect. Derycke $e t a l^{8}$ reported that the exposure to air or oxygen induced an increase in the work function of the gold electrode, which resulted in ambipolar behavior. Indeed, we observe a similar effect of ambient air on our DWNT-FET which shows an increase in the $p$-channel transport as well as a decrease in the $n$-channel conduction, leading to asymmetric $I_{\mathrm{ds}}-V_{g}$ patterns. It is noticed that the $I_{\mathrm{ds}}-V_{g}$ curve for our DWNT-FET shows a symmetriclike transport behavior under the high-vacuum condition, but this symmetry goes away as the system is slowly exposed to air.

Although a part of our results is consistent with previous works, ${ }^{7,8}$ the unipolar behavior of DWNT-FET in ambient air, as shown in Fig. 1, needs more comprehensible arguments. It is reasonable to assume that there are significant molecular adsorptions on the wall of CNT as well as on the surface of metal electrode. Provided that the lowest unoccupied molecular orbital (LUMO) levels of the adsorbators sit somewhere in the gap of the CNT, electrons would occupy the molecular levels under a positive gate voltage, as shown in Figs. 3(a) and 3(b). As a result, the electrostatic potential generated by the negatively charged adsorbators screens the gate field and, consequently, suppresses the electron channel formation. The nanotube bands in the channel region would resist a downshift because of the presence of charged adsorbators. Two upward arrows in Fig. 3(b) describe the effect of such an electrostatic potential generated by the charged adsorbators. On the other hand, conduction bands of a clean 

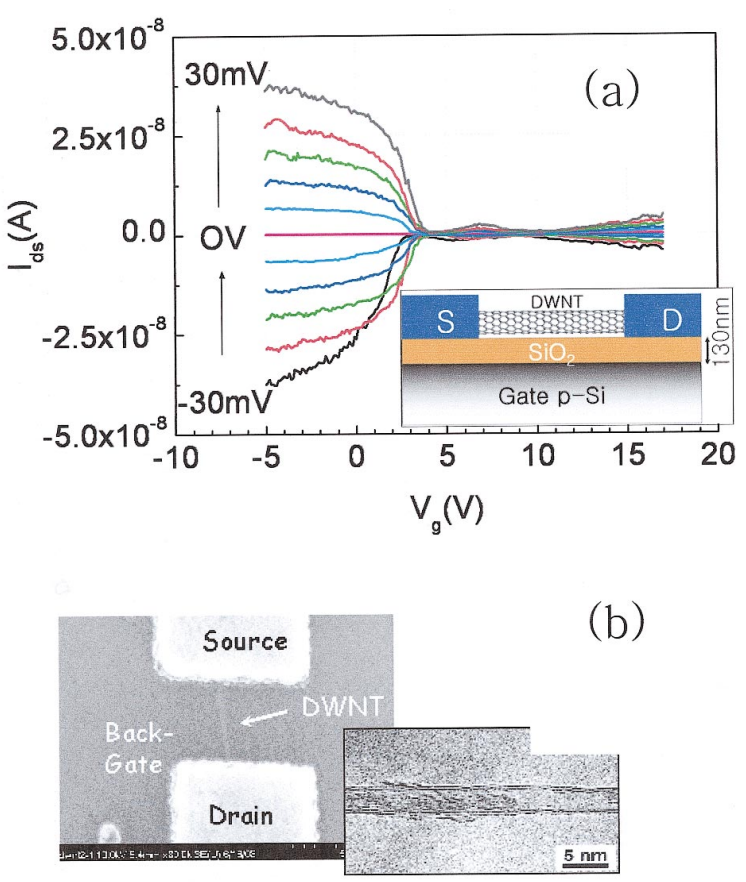

FIG. 1. (Color) (a) The upper panel shows $I_{\mathrm{ds}}-V_{g}$ curves of the DWNT-FET under the influence of ambient air condition with various source-drain voltages. A schematic drawing of the device is given in inset. (b) Scanning electron microscopy figure of the DWNT-FET and high-resolution transmission electron microscopy figure of DWNT used for this work.

nanotube shift down allowing electron current under such a positive gate voltage, as shown in Fig. 3(c). It is noticed that the $V_{g}$ difference between the onset voltage of $p$-channel and the bumps in Figs. 1 and 2 is about $3 \mathrm{~V}$, which is similar to the off-state range of the DWNT-FET under a vacuum. Thus, based on this theory, the small bumps in the $I_{\mathrm{ds}}-V_{g}$ curves and subsequent small traces of the current tailing in the positive side of $V_{g}$ would be precursors of $n$ channels in the otherwise ambipolar DWNT-FET.

We performed ab initio density-functional (DFT) calculations using the basis of plane-wave set with the cutoff energy of $500 \mathrm{eV} .^{9,10}$ The ultrasoft pseudopotentials are implemented for the ionic potentials, ${ }^{11}$ and the generalized gradient approximation (GGA) is used for the exchangecorrelation potential. ${ }^{12}$ Since it is well known that the influences of $\mathrm{O}_{2}$ and $\mathrm{H}_{2} \mathrm{O}$ are significant on the device performance of CNT-FET, ${ }^{7,8,13}$ it is necessary to investigate the effect of these molecules on the CNT. The $(10,0)$ CNT,

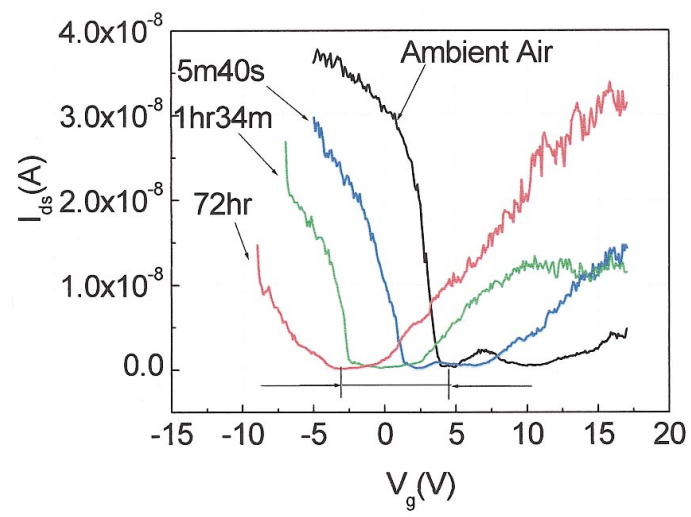

FIG 2 (Color) Transfer charecteristics of DWNT.FET under ambient air vacuum of $3 \times 10^{-6}$ Torr, and at intermediate pumping stages.

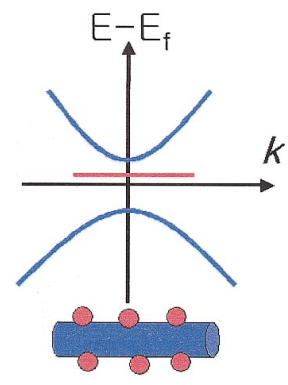

(a)

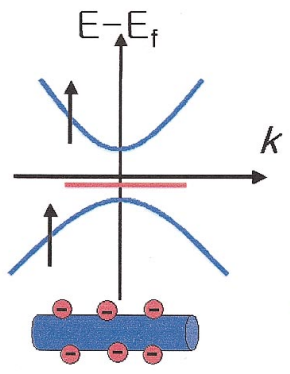

(b)

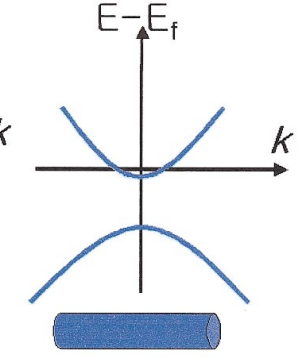

(c)
FIG. 3. (Color) Schematic band structure of the carbon nanotube (blue) and the LUMO level of the adsorbators (red), with respect to the Fermi level.

whose band gap is found to be $0.7 \mathrm{eV}$, is exploited as an example for a typical semiconducting CNT. Despite the fact that commonly synthesized nanotubes may have a larger diameter than the $(10,0)$ nanotube, the results, which will be discussed in this paragraph, can be applied for such large diameter semiconducting nanotubes. Twice the minimal unit cell of the zigzag nanotube $(\approx 8.49 \AA)$ is used for the unit cell along the axial direction of the CNT. Along the perpendicular direction to the axis, the nanotube is separated from its replica with a large vacuum region of about $10 \AA$. As shown in Fig. 4, the fully relaxed $\mathrm{O}_{2}$ position is located in between two $\mathrm{C}-\mathrm{C}$ bonds with a binding energy of $0.013 \mathrm{eV}$, which is in an overall agreement with the previous works. ${ }^{14,15}$ The dispersion forces are not dealt within the DFT calculations. However, a proper consideration of dispersion forces would increase the binding energy about $0.1 \mathrm{eV}$ over that of the GGA value. ${ }^{14}$ In fact, we observe that the LUMO level of oxygen lies somewhere in the gap of the CNT. Since the oxygen adsorption on the surface of nanotube is a weak physisorption with negligible charge transfer, ${ }^{15,16}$ two half-filled $p p \pi^{*}$ states should be around the Fermi level of the CNT. In other words, there should be unoccupied molecular levels at or just above the Fermi level, irrespective of the diameter of the semiconducting CNT.

The effect of oxygen adsorption on CNT has been controversial. An earlier study suggested that the binding energy of $\mathrm{O}_{2}$ on $\mathrm{CNT}$ wall is strong, and thus the substantial charge transfer results in the hole doping of the CNT. ${ }^{17}$ Subsequent studies have declined the possibility of the $\mathrm{O}_{2}$-induced hole doping. ${ }^{14-16}$ However, we have found that the LUMO level of $\mathrm{O}_{2}$ adsorbators could provide the charge trapping centers,

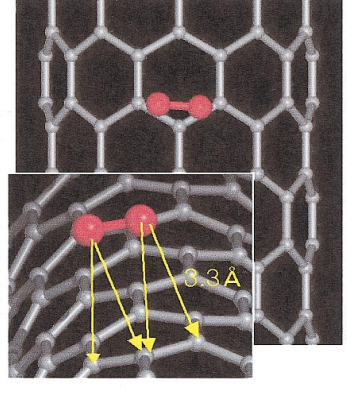

(a)

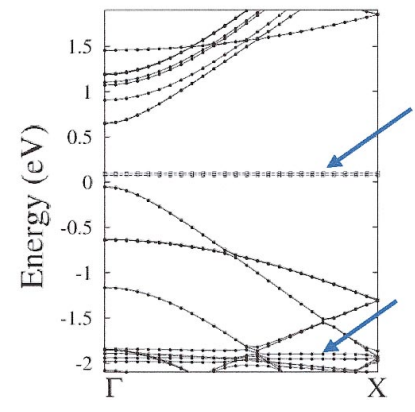

(b)
FIG. 4. (Color) Adsorption geometry of oxygen molecule on the wall of the $(10,0)$ nanotube and $a b$ initio band structure the oxygen adsorbed nanotube system. Arrows in (b) indicate oxygen molecular states. 
irrespective of the possibility of a practical hole doping by the oxygen adsorption. These charge trapping centers could screen the electric field from the positively biased gate, resulting in the suppression of electron channel formation.

We performed the same calculation for the wateradsorbed CNT, adopting the adsorption geometry discussed in literature. ${ }^{18}$ It is found that the LUMO level of $\mathrm{H}_{2} \mathrm{O}$ is about $4 \mathrm{eV}$ above the Fermi level of the CNT. Thus, it is believed that the effect of water vapor on the aforementioned suppression of the $n$-channel formation is insignificant. ${ }^{19}$

In summary, we observed a unipolar behavior of DWNTFET under ambient air. We suggest that electrons trapped by the adsorbators, whose LUMO level is in the band gap of the CNT, suppress the $n$-channel formation on the positive gate voltage, leading to a unipolar transfer characteristic. Through $a b$ initio density-functional calculations, we concluded that the oxygen molecule could be the most probable candidate for such an adsorbator, which induces the conversion of DWNT-FET into a unipolar behavior.

The authors thank J. R. Kim and J. J. Kim at Chonbuk National University for their cooperation. This research project was supported by the Terra-level Nano Devices, Korea National Research Program.

\footnotetext{
${ }^{1}$ S. Iijima, Nature (London) 354, 56 (1991).

${ }^{2}$ A. Javey, H. Kim, M. Brink, Q. Wang, A. Ural, J. Guo, P. Mcintyre, P. Mceuen, M. Lundsrtrom, and H. Dai, Nat. Mater. 1, 241 (2002).
}

${ }^{3}$ S. Frank, P. Poncharal, Z. Wang, and W. Heer, Science 280, 1744 (1998).

${ }^{4}$ R. Martel, T. Schmidt, H. R. Shea, T. Hertel, and P. Avouris, Appl. Phys. Lett. 73, 2447 (1998).

${ }^{5}$ P. G. Collins, M. S. Arnold, and P. Avouris, Science 292, 706 (2001); T. Shimada, T. Okazaki, R. Taniguchi, T. Sugai, H. Shinohara, K. Suenaga, Y. Ohno, S. Mizuno, S. Kishimoto, and T. Mizutani, Appl. Phys. Lett. 81, 4067 (2002).

${ }^{6}$ T. Shimada, T. Sugai, Y. Ohno, S. Kishimoto, T. Mizutani, H. Yoshida, T. Okazaki, and H. Shinohara, Appl. Phys. Lett. 84, 2412 (2004).

${ }^{7}$ S. Heinze, J. Tersoff, R. Martel, V. Derycke, J. Appenzeller, and P. Avouris, Phys. Rev. Lett. 89, 106801 (2002).

${ }^{8}$ V. Derycke, R. Martel, J. Appenzeller, and P. Avouris, Appl. Phys. Lett. 80, 2773 (2002).

${ }^{9}$ P. Hohenberg and W. Kohn, Phys. Rev. 136, B864 (1964); W. Kohn and L. J. Sham, Phys. Rev. 140, A1133 (1965).

${ }^{10}$ J. Ihm, A. Zunger, and M. L. Cohen, J. Phys. C 12, 4409 (1979).

${ }^{11}$ D. Vanderbilt, Phys. Rev. B 41, 7892 (1990).

${ }^{12}$ J. P. Perdew, K. Burke, and M. Ernzerhof, Phys. Rev. Lett. 77, 3865 (1996).

${ }^{13}$ W. Kim, A. Javey, O. Vermesh, Q. Wang, Y. Li, and H. Dai, Nano Lett. 3, 193 (2003).

${ }^{14}$ S. Dag, O. Gulsere, T. Yildirim, and S. Ciraci, Phys. Rev. B 67, 165424 (2003).

${ }^{15}$ P. Giannozzi, R. Car, and G. Scoles, J. Chem. Phys. 118, 1003 (2003).

${ }^{16}$ H. Ulbricht, G. Moos, and T. Hertel, Phys. Rev. B 66, 075404 (2002).

${ }^{17}$ S.-H. Jhi, S. G. Louie, and M. L. Cohen, Phys. Rev. Lett. 85, 1710 (2000).

${ }^{18}$ R. Pati, Y. Zhang, and S. K. Nayak, Appl. Phys. Lett. 81, 2638 (2002).

${ }^{19}$ Because the highest occupied molecular level (HOMO)-LUMO gap in the DFT is not so rigorous, we performed TDDFT calculations for $\mathrm{H}_{2} \mathrm{O}$ using Gaussian package [Gaussian 03, Revision B.01, Gaussian, Inc., Pittsburgh, PA, 2003]. The HOMO-LUMO gap of $\mathrm{H}_{2} \mathrm{O}$ is found to be 6.4 and $7.5 \mathrm{eV}$ in DFT and TDDFT, respectively. Thus, the DFT artifact would not affect our model discussed here. 